\title{
Generalized Antiperiodic Boundary Value Problems for the Fractional Differential Equation with p-Laplacian Operator
}

\author{
Zhi-Wei Lv ${ }^{1,2}$ and Xu-Dong Zheng ${ }^{2}$ \\ ${ }^{1}$ Department of Mathematics, Zhengzhou University, Zhengzhou, Henan 450001, China \\ ${ }^{2}$ Department of Mathematics and Physics, Anyang Institute of Technology, Anyang, Henan 455000, China
}

Correspondence should be addressed to Zhi-Wei Lv; sdlllzw@mail.ustc.edu.cn

Received 3 February 2013; Accepted 12 March 2013

Academic Editor: Hua Su

Copyright (C) 2013 Z.-W. Lv and X.-D. Zheng. This is an open access article distributed under the Creative Commons Attribution License, which permits unrestricted use, distribution, and reproduction in any medium, provided the original work is properly cited.

We discuss the existence of solutions about generalized antiperiodic boundary value problems for the fractional differential equation with p-Laplacian operator $\phi_{p}\left({ }^{c} D_{0+}^{\alpha} u(t)\right)=f\left(t, u(t), u^{\prime}(t)\right), 0<t<T, 1<\alpha \leq 2, u(0)+(-1)^{\theta} a u(T)=0$, ${ }^{c} D_{0+}^{\beta} u(0)+(-1)^{\theta} b^{c} D_{0+}^{\beta} u(T)=\lambda, 0<\beta<1$, where ${ }^{c} D_{0+}^{\alpha}$ is the Caputo fractional derivative, $\theta=0,1, a>0, a \neq 1, b>0$ and $\phi_{p}(s)=|s|^{p-2} s, p>1, \phi_{p}^{-1}=\phi_{q}, 1 / p+1 / q=1$. Our results are based on fixed point theorem and contraction mapping principle. Furthermore, three examples are also given to illustrate the results.

\section{Introduction}

Fractional differential equations arise in various areas of science and engineering, such as physics, mechanics, chemistry, and engineering. The fractional order models become more realistic and practical than the classical integer models. Due to their applications, fractional differential equations have gained considerable attentions; one can see [1-14] and references therein.

Anti-periodic boundary value problems occur in the mathematical modeling of a variety of physical processes. Anti-periodic problems constitute an important class of boundary value problems and have received considerable attention (see [15-19]).

In [20], Zhang considered the existence and multiplicity results of positive solutions for the following boundary value problem of fractional differential equation:

$$
\begin{gathered}
{ }^{c} D_{0+}^{p} u(t)=f(t, u(t)), \quad 0<t<1, \\
u(0)+u^{\prime}(0)=0, \quad u(1)+u^{\prime}(1)=0,
\end{gathered}
$$

where $1<p \leq 2$ is a real number, ${ }^{c} D^{q}$ is the Caputo fractional derivative, and $f:[0,1] \times[0,+\infty) \rightarrow[0,+\infty)$ is continuous.

In [15], the authors discussed some existence results for the following anti-periodic boundary value problem for fractional differential equations:

$$
\begin{gathered}
{ }^{c} D^{q} x(t)=f(t, x(t)), \quad t \in[0, T], T>0,3<q \leq 4, \\
x(0)=-x(T), \quad x^{\prime}(0)=-x^{\prime}(T), \\
x^{\prime \prime}(0)=-x^{\prime \prime}(T), \quad x^{\prime \prime \prime}(0)=-x^{\prime \prime \prime}(T),
\end{gathered}
$$

where ${ }^{c} D^{q}$ is the Caputo fractional derivative of order $q$; $f$ is a given continuous function.

In [16], the authors investigated the following antiperiodic boundary value problem for higher-order fractional differential equations:

$$
{ }^{c} D^{q} x(t)=f(t, x(t)), \quad t \in[0, T], T>0,3<q \leq 4,
$$




$$
\begin{gathered}
x(0)=-x(T), \quad x^{\prime}(0)=-x^{\prime}(T), \\
x^{\prime \prime}(0)=-x^{\prime \prime}(T), \quad x^{\prime \prime \prime}(0)=-x^{\prime \prime \prime}(T),
\end{gathered}
$$

where ${ }^{c} D^{q}$ is the Caputo fractional derivative of order $q$; $f$ is a given continuous function.

In [17], the authors investigated a class of anti-periodic boundary value problem of fractional differential equations

$$
\begin{gathered}
{ }^{c} D^{q} x(t)=f(t, x(t)), \quad t \in[0, T], T>0,1<q \leq 2, \\
x(0)=-x(T), \quad{ }^{c} D_{0+}^{p} x(0)=-{ }^{c} D_{0+}^{p} u(T), \quad 0<p<1,
\end{gathered}
$$

where ${ }^{c} D^{q}$ is the Caputo fractional derivative of order $q$; $f$ is a given continuous function.

In this paper, we discuss the existence of solutions about generalized anti-periodic boundary value problems for the fractional differential equation with p-Laplacian operator

$$
\begin{gathered}
\phi_{p}\left({ }^{c} D_{0+}^{\alpha} u(t)\right)=f\left(t, u(t), u^{\prime}(t)\right), \quad 0<t<T, 1<\alpha \leq 2, \\
u(0)+(-1)^{\theta} a u(T)=0, \\
{ }^{c} D_{0+}^{\beta} u(0)+(-1)^{\theta} b^{c} D_{0+}^{\beta} u(T)=\lambda, \quad 0<\beta<1,
\end{gathered}
$$

where ${ }^{c} D_{0+}^{\alpha}$ is the Caputo fractional derivative, $\theta=0,1, a>$ $0, a \neq 1, b>0$, and $\phi_{p}(s)=|s|^{p-2} s, p>1, \phi_{p}^{-1}=\phi_{q},(1 / p)+$ $(1 / q)=1$.

If we take $a=b=1, \theta=\lambda=0$ and $p=2$, then the problem (5) becomes the problem studied in [17]. In this paper, we let $a \neq 1$.

This paper is organized as follows. In Section 2, we present some background materials and preliminaries. Section 3 deals with some existence results. In Section 4, three examples are given to illustrate the results.

\section{Background Materials and Preliminaries}

Definition 1 (see [21]). The fractional integral of order $\alpha$ with the lower limit $t_{0}$ for a function $f$ is defined as

$$
I^{\alpha} f(t)=\frac{1}{\Gamma(\alpha)} \int_{t_{0}}^{t}(t-s)^{\alpha-1} f(s) d s, \quad t>t_{0}, \alpha>0,
$$

where $\Gamma$ is the gamma function.

Definition 2 (see [21]). Caputo's derivative of order $q$ with the lower limit $t_{0}$ for a function $f$ can be written as

$$
\begin{array}{r}
{ }^{c} D^{q} f(t)=\frac{1}{\Gamma(n-q)} \int_{t_{0}}^{t}(t-s)^{n-q-1} f^{(n)}(s) d s, \\
t>t_{0}, q>0, n=[q]+1 .
\end{array}
$$

Lemma 3 (see $[22])$. Assume that $u \in C(0,1) \cap L(0,1)$ with a fractional derivative of order $\alpha>0$ that belongs to $C(0,1) \cap$ $L(0,1)$. Then

$$
I_{0+}^{\alpha}{ }^{c} D_{0+}^{\alpha} u(t)=u(t)+c_{0}+c_{1} t+c_{2} t+\cdots+c_{n-1} t^{n-1},
$$

Lemma 4. Let $y \in C[0,1]$. Then the fractional differential equation

$$
\begin{gathered}
\phi_{p}\left({ }^{c} D_{0+}^{\alpha} u(t)\right)=y(t), \quad 0<t<T, 1<\alpha \leq 2, \\
u(0)+(-1)^{\theta} a u(T)=0, \\
{ }^{c} D_{0+}^{\beta} u(0)+(-1)^{\theta} b^{c} D_{0+}^{\beta} u(T)=\lambda
\end{gathered}
$$

has a unique solution which is given by

$$
\begin{aligned}
u(t)= & \frac{1}{\Gamma(\alpha)} \int_{0}^{t}(t-s)^{\alpha-1} y(s) d s \\
& -\frac{a}{\left[(-1)^{\theta}+a\right] \Gamma(\alpha)} \int_{0}^{T}(T-s)^{\alpha-1} y(s) d s \\
& +\frac{a \Gamma(2-\beta)}{\left[(-1)^{\theta}+a\right] T^{-\beta} \Gamma(\alpha-\beta)} \int_{0}^{T}(T-s)^{\alpha-\beta-1} y(s) d s \\
& -\frac{a \Gamma(2-\beta)}{\left[1+(-1)^{\theta} a\right] b T^{-\beta}} \\
& +t\left[-\frac{\Gamma(2-\beta)}{T^{1-\beta} \Gamma(\alpha-\beta)} \int_{0}^{T}(T-s)^{\alpha-\beta-1} y(s) d s\right. \\
& \left.+\frac{(-1)^{\theta} \Gamma(2-\beta)}{b T^{1-\beta}} \lambda\right] .
\end{aligned}
$$

Proof. From Lemma 3, we have

$$
\begin{aligned}
u(t)= & I_{0+}^{\alpha}\left(\phi_{q}(y(t))\right)+c_{0}+c_{1} t \\
= & \frac{1}{\Gamma(\alpha)} \int_{0}^{t}(t-s)^{\alpha-1} \phi_{q}(y(s)) d s+c_{0}+c_{1} t \\
{ }^{c} D_{0+}^{\beta} u(t)= & I_{0+}^{(\alpha-\beta)}\left(\phi_{q}(y(t))\right)+c_{1}{ }^{c} D_{0+}^{\beta} t \\
= & \frac{1}{\Gamma(\alpha-\beta)} \int_{0}^{t}(t-s)^{\alpha-\beta-1} \phi_{q}(y(s)) d s \\
& +c_{1} \frac{t^{1-\beta}}{\Gamma(2-\beta)} .
\end{aligned}
$$

Thus,

$$
\begin{gathered}
u(0)=c_{0}, \\
u(T)=\frac{1}{\Gamma(\alpha)} \int_{0}^{T}(T-s)^{\alpha-1} \phi_{q}(y(s)) d s+c_{0}+c_{1} T, \\
{ }^{c} D_{0+}^{\beta} u(0)=0, \\
+c_{1} \frac{T^{1-\beta}}{\Gamma(2-\beta)} .
\end{gathered}
$$


By ${ }^{c} D_{0+}^{\beta} u(0)+(-1)^{\theta} b^{c} D_{0+}^{\beta} u(T)=\lambda$, we have

$$
\begin{aligned}
c_{1}= & -\frac{\Gamma(2-\beta)}{T^{1-\beta} \Gamma(\alpha-\beta)} \int_{0}^{T}(T-s)^{\alpha-\beta-1} \phi_{q}(y(s)) d s \\
& +\frac{(-1)^{\theta} \Gamma(2-\beta)}{b T^{1-\beta}} \lambda .
\end{aligned}
$$

Using the boundary condition $u(0)+(-1)^{\theta} a u(T)=0$ and (13), we obtain

$$
\begin{aligned}
c_{0}= & -\frac{a}{\left[(-1)^{\theta}+a\right] \Gamma(\alpha)} \int_{0}^{T}(T-s)^{\alpha-1} \phi_{q}(y(s)) d s \\
& +\frac{a \Gamma(2-\beta)}{\left[(-1)^{\theta}+a\right] T^{-\beta} \Gamma(\alpha-\beta)} \int_{0}^{T}(T-s)^{\alpha-\beta-1} \phi_{q}(y(s)) d s \\
& -\frac{a \Gamma(2-\beta)}{\left[1+(-1)^{\theta} a\right] b T^{-\beta}} \lambda .
\end{aligned}
$$

Thus,

$$
\begin{aligned}
u(t)= & \frac{1}{\Gamma(\alpha)} \int_{0}^{t}(t-s)^{\alpha-1} \phi_{q}(y(s)) d s \\
& -\frac{a}{\left[(-1)^{\theta}+a\right] \Gamma(\alpha)} \int_{0}^{T}(T-s)^{\alpha-1} \phi_{q}(y(s)) d s \\
& +\frac{a \Gamma(2-\beta)}{\left[(-1)^{\theta}+a\right] T^{-\beta} \Gamma(\alpha-\beta)} \\
& \times \int_{0}^{T}(T-s)^{\alpha-\beta-1} \phi_{q}(y(s)) d s \\
& -\frac{a \Gamma(2-\beta)}{\left[1+(-1)^{\theta} a\right] b T^{-\beta}} \lambda \\
+ & t\left[-\frac{\Gamma(2-\beta)}{T^{1-\beta} \Gamma(\alpha-\beta)}\right. \\
& \times \int_{0}^{T}(T-s)^{\alpha-\beta-1} \phi_{q}(y(s)) d s \\
& \left.\quad+\frac{(-1)^{\theta} \Gamma(2-\beta)}{b T^{1-\beta}} \lambda\right] .
\end{aligned}
$$

\section{Main Results}

Let $X=C^{1}([0,1], R)$ denote the Banach space of continuous functions $u(t)$ and $u^{\prime}(t)$ from $[0,1] \rightarrow R$ endowed with the norm defined by

$$
\|u\|_{1}=\max \left\{\|u\|,\left\|u^{\prime}\right\|\right\},
$$

where

$$
\|u\|=\sup _{0 \leq t \leq 1}|u(t)|, \quad\left\|u^{\prime}\right\|=\sup _{0 \leq t \leq 1}\left|u^{\prime}(t)\right| .
$$

Define an operator $\mathrm{F}: X \rightarrow X$ as

$$
\begin{aligned}
\mathrm{F} u(t)= & \frac{1}{\Gamma(\alpha)} \int_{0}^{t}(t-s)^{\alpha-1} \phi_{q}\left(f\left(s, u(s), u^{\prime}(s)\right)\right) d s \\
& -\frac{a}{\left[(-1)^{\theta}+a\right] \Gamma(\alpha)} \\
& \times \int_{0}^{T}(T-s)^{\alpha-1} \phi_{q}\left(f\left(s, u(s), u^{\prime}(s)\right)\right) d s \\
& +\frac{a \Gamma(2-\beta)}{\left[(-1)^{\theta}+a\right] T^{-\beta} \Gamma(\alpha-\beta)} \\
& \times \int_{0}^{T}(T-s)^{\alpha-\beta-1} \phi_{q}\left(f\left(s, u(s), u^{\prime}(s)\right)\right) d s \\
& -\frac{a \Gamma(2-\beta)}{\left[1+(-1)^{\theta} a\right] b T^{-\beta}} \lambda \\
& +t \quad-\frac{\Gamma(2-\beta)}{T^{1-\beta} \Gamma(\alpha-\beta)} \\
& \quad \times \int_{0}^{T}(T-s)^{\alpha-\beta-1} \phi_{q}\left(f\left(s, u(s), u^{\prime}(s)\right)\right) d s \\
& \left.+\frac{(-1)^{\theta} \Gamma(2-\beta)}{b T^{1-\beta}} \lambda\right] .
\end{aligned}
$$

From (18), we conclude that

$$
\begin{aligned}
\mathrm{F}^{\prime} u(t)= & \frac{1}{\Gamma(\alpha-1)} \int_{0}^{t}(t-s)^{\alpha-2} \phi_{q}\left(f\left(s, u(s), u^{\prime}(s)\right)\right) d s \\
& -\frac{\Gamma(2-\beta)}{T^{1-\beta} \Gamma(\alpha-\beta)} \\
& \times \int_{0}^{T}(T-s)^{\alpha-\beta-1} \phi_{q}\left(f\left(s, u(s), u^{\prime}(s)\right)\right) d s \\
& +\frac{(-1)^{\theta} \Gamma(2-\beta)}{b T^{1-\beta}} \lambda .
\end{aligned}
$$

Then (5) has a solution if and only if the operator $F$ has a fixed point.

Theorem 5. Let $f:[0, T] \times R_{+} \times R_{+} \rightarrow R_{+}$be continuous. Assume that $f$ meets the following condition: there exist $d \geq 0$, $\gamma \geq 0$ such that

$$
f\left(t, x(t), x^{\prime}(t)\right) \leq \gamma \phi_{p}(|x(t)|), \quad \text { for } t \in[0, T],\|x\|_{1} \leq d,
$$




$$
\begin{gathered}
\phi_{q}(\gamma) \leq \frac{1}{2}\left(\frac{T^{\alpha-1}}{\Gamma(\alpha)}+\frac{T^{\alpha}}{\Gamma(\alpha+1)}+\frac{a T^{\alpha}}{|1-a| \Gamma(\alpha+1)}\right. \\
+\frac{a T^{\alpha} \Gamma(2-\beta)}{|1-a| \Gamma(\alpha-\beta+1)}+\frac{T^{\alpha} \Gamma(2-\beta)}{\Gamma(\alpha-\beta+1)} \\
\left.+\frac{T^{\alpha-1} \Gamma(2-\beta)}{\Gamma(\alpha-\beta+1)}\right)^{-1} .
\end{gathered}
$$

Then the problem (5) has at least one solution on $[0, T]$ for

$$
0 \leq \lambda \leq \frac{d}{2}\left(\frac{a \Gamma(2-\beta)}{|1-a| b T^{-\beta}}+\frac{\Gamma(2-\beta)}{b T^{-\beta}}+\frac{\Gamma(2-\beta)}{b T^{1-\beta}}\right)^{-1} .
$$

Proof. From $f\left(t, u(t), u^{\prime}(t)\right) \in C\left([0,1] \times R_{+} \times R_{+}, R_{+}\right)$, we know that $T$ is continuous.

Let

$$
B_{d}=\left\{u \mid\|u\|_{1} \leq d, u \in X\right\}
$$

For $u \in B_{d}$, we have

$$
\begin{aligned}
|\mathrm{F} u(t)| \leq & \frac{1}{\Gamma(\alpha)} \int_{0}^{t}(t-s)^{\alpha-1}\left|\phi_{q}\left(f\left(s, u(s), u^{\prime}(s)\right)\right)\right| d s \\
& +\frac{a}{\left|(-1)^{\theta}+a\right| \Gamma(\alpha)} \\
& \times \int_{0}^{T}(T-s)^{\alpha-1}\left|\phi_{q}\left(f\left(s, u(s), u^{\prime}(s)\right)\right)\right| d s \\
& +\frac{a \Gamma(2-\beta)}{\left|(-1)^{\theta}+a\right| T^{-\beta} \Gamma(\alpha-\beta)} \\
& \times \int_{0}^{T}(T-s)^{\alpha-\beta-1}\left|\phi_{q}\left(f\left(s, u(s), u^{\prime}(s)\right)\right)\right| d s \\
& +\left|\frac{a \Gamma(2-\beta)}{\left[1+(-1)^{\theta} a\right] b T^{-\beta}} \lambda\right| \\
& +t \mid \frac{\Gamma(2-\beta)}{T^{1-\beta} \Gamma(\alpha-\beta)} \\
& \times \int_{0}^{T}(T-s)^{\alpha-\beta-1}\left|\phi_{q}\left(f\left(s, u(s), u^{\prime}(s)\right)\right)\right| d s \\
& \left.+\left|\frac{(-1)^{\theta} \Gamma(2-\beta)}{b T^{1-\beta}} \lambda\right|\right]
\end{aligned}
$$

$$
\begin{aligned}
& \leq \frac{1}{\Gamma(\alpha)} \int_{0}^{t}(t-s)^{\alpha-1} \phi_{q}(\gamma)|u(s)| d s \\
& +\frac{a}{|1-a| \Gamma(\alpha)} \int_{0}^{T}(T-s)^{\alpha-1} \phi_{q}(\gamma)|u(s)| d s \\
& +\frac{a \Gamma(2-\beta)}{|1-a| T^{-\beta} \Gamma(\alpha-\beta)} \\
& \times \int_{0}^{T}(T-s)^{\alpha-\beta-1} \phi_{q}(\gamma)|u(s)| d s \\
& +\frac{a \Gamma(2-\beta)}{|1-a| b T^{-\beta}} \lambda \\
& +t\left[\frac{\Gamma(2-\beta)}{T^{1-\beta} \Gamma(\alpha-\beta)}\right. \\
& \times \int_{0}^{T}(T-s)^{\alpha-\beta-1} \phi_{q}(\gamma)|u(s)| d s \\
& \left.+\frac{\Gamma(2-\beta)}{b T^{1-\beta}} \lambda\right] \\
& \leq\left(\frac{T^{\alpha} \phi_{q}(\gamma)}{\Gamma(\alpha+1)}+\frac{a T^{\alpha} \phi_{q}(\gamma)}{|1-a| \Gamma(\alpha+1)}\right. \\
& +\frac{a T^{\alpha} \Gamma(2-\beta) \phi_{q}(\gamma)}{|1-a| \Gamma(\alpha-\beta+1)} \\
& \left.+\frac{T^{\alpha} \Gamma(2-\beta) \phi_{q}(\gamma)}{\Gamma(\alpha-\beta+1)}\right)\|u\|_{1} \\
& +\frac{a \Gamma(2-\beta)}{|1-a| b T^{-\beta}} \lambda+\frac{\Gamma(2-\beta)}{b T^{-\beta}} \lambda,
\end{aligned}
$$$$
\left|\mathrm{F}^{\prime} u(t)\right| \leq \frac{1}{\Gamma(\alpha-1)}
$$$$
\times \int_{0}^{t}(t-s)^{\alpha-2}\left|\phi_{q}\left(f\left(s, u(s), u^{\prime}(s)\right)\right)\right| d s
$$$$
+\frac{\Gamma(2-\beta)}{T^{1-\beta} \Gamma(\alpha-\beta)}
$$$$
\times \int_{0}^{T}(T-s)^{\alpha-\beta-1}\left|\phi_{q}\left(f\left(s, u(s), u^{\prime}(s)\right)\right)\right| d s
$$$$
+\frac{\Gamma(2-\beta)}{b T^{1-\beta}} \lambda
$$$$
\leq \frac{1}{\Gamma(\alpha-1)} \int_{0}^{t}(t-s)^{\alpha-2} \phi_{q}(\gamma)|u(s)| d s
$$$$
+\frac{\Gamma(2-\beta)}{T^{1-\beta} \Gamma(\alpha-\beta)} \int_{0}^{T}(T-s)^{\alpha-\beta-1} \phi_{q}(\gamma)|u(s)| d s
$$$$
+\frac{\Gamma(2-\beta)}{b T^{1-\beta}} \lambda
$$ 


$$
\begin{aligned}
\leq & \left(\frac{T^{\alpha-1} \phi_{q}(\gamma)}{\Gamma(\alpha)}+\frac{T^{\alpha-1} \Gamma(2-\beta) \phi_{q}(\gamma)}{\Gamma(\alpha-\beta+1)}\right)\|u\|_{1} \\
& +\frac{\Gamma(2-\beta)}{b T^{1-\beta}} \lambda .
\end{aligned}
$$

This, together with (21) and (22), yields that

$$
\|\mathrm{F} u(t)\|_{1} \leq d .
$$

Hence, $\mathrm{F}\left(B_{d}\right)$ is uniformly bounded.

Next we show that $\mathrm{F}$ is equicontinuous.

For any $0 \leq t_{1} \leq t_{2} \leq 1, u \in B_{d}$, we have

$$
\begin{aligned}
& \left|\mathrm{F} u\left(t_{2}\right)-\mathrm{Fu}\left(t_{1}\right)\right| \\
& \leq \mid \frac{1}{\Gamma(\alpha)} \int_{0}^{t_{2}}\left(t_{2}-s\right)^{\alpha-1} \phi_{q}\left(f\left(s, u(s), u^{\prime}(s)\right)\right) d s \\
& \quad-\frac{1}{\Gamma(\alpha)} \int_{0}^{t_{1}}\left(t_{1}-s\right)^{\alpha-1} \phi_{q}\left(f\left(s, u(s), u^{\prime}(s)\right)\right) d s \mid \\
& +\left[\frac{\Gamma(2-\beta)}{T^{1-\beta} \Gamma(\alpha-\beta)}\right. \\
& \quad \times \int_{0}^{T}(T-s)^{\alpha-\beta-1} \phi_{q}\left(f\left(s, u(s), u^{\prime}(s)\right)\right) d s \\
& \quad+\frac{1}{\Gamma(\alpha)} \int_{0}^{t_{1}}\left|\left(t_{2}-s\right)^{\alpha-1}-\left(t_{1}-s\right)^{\alpha-1}\right| \phi_{q}(\gamma)|u(s)| d s \\
& +\frac{1}{\Gamma(\alpha)} \int_{t_{1}}^{t_{2}}\left(t_{1}-s\right)^{\alpha-1} \phi_{q}(\gamma)|u(s)| d s \\
& +\left[\frac{\Gamma(2-\beta)}{T^{1-\beta} \Gamma(\alpha-\beta)} \int_{0}^{T}(T-s)^{\alpha-\beta-1} \phi_{q}(\gamma)|u(s)| d s\right. \\
& +\frac{\phi_{q}(\gamma) d}{\Gamma(\alpha)} \int_{0}^{t_{1}}\left|\left(t_{2}-s\right)^{\alpha-1}-\left(t_{1}-s\right)^{\alpha-1}\right| d s \\
& +\frac{\phi_{q}(\gamma) d}{\Gamma(\alpha)} \int_{t_{1}}^{t_{2}}\left(t_{1}-s\right)^{\alpha-1} d s \\
& \left.\quad+\frac{T^{\alpha-1} \phi_{q}(\gamma) d \Gamma(2-\beta)}{\Gamma T^{1-\beta}} \lambda\right]\left(t_{2}-t_{1}\right)
\end{aligned}
$$

$$
\begin{aligned}
\left|\mathrm{F}^{\prime} u\left(t_{2}\right)-\mathrm{F}^{\prime} u\left(t_{1}\right)\right| & \mid \\
\leq & \mid \frac{1}{\Gamma(\alpha-1)} \int_{0}^{t_{2}}\left(t_{2}-s\right)^{\alpha-2} \phi_{q}\left(f\left(s, u(s), u^{\prime}(s)\right)\right) d s \\
& \quad-\frac{1}{\Gamma(\alpha-1)} \int_{0}^{t_{1}}\left(t_{1}-s\right)^{\alpha-2} \phi_{q}\left(f\left(s, u(s), u^{\prime}(s)\right)\right) d s \mid \\
\leq & \frac{1}{\Gamma(\alpha-1)} \int_{0}^{t_{1}}\left|\left(t_{2}-s\right)^{\alpha-1}-\left(t_{1}-s\right)^{\alpha-1}\right| \phi_{q}(\gamma)|u(s)| d s \\
& +\frac{1}{\Gamma(\alpha-1)} \int_{t_{1}}^{t_{2}}\left(t_{1}-s\right)^{\alpha-2} \phi_{q}(\gamma)|u(s)| d s \\
\leq & \frac{\phi_{q}(\gamma) d}{\Gamma(\alpha-1)} \int_{0}^{t_{1}}\left|\left(t_{2}-s\right)^{\alpha-2}-\left(t_{1}-s\right)^{\alpha-2}\right| d s \\
& +\frac{\phi_{q}(\gamma) d}{\Gamma(\alpha-1)} \int_{t_{1}}^{t_{2}}\left(t_{1}-s\right)^{\alpha-2} d s .
\end{aligned}
$$

Thus, we conclude that $\mathrm{F}$ is equicontinuous on $B_{d}$, and

$$
\mathrm{F}: B_{d} \longrightarrow B_{d} \text { is completely continuous. }
$$

By Schauder fixed point theorem we know that there exists a solution for the boundary value problem (5).

Theorem 6. Let $f:[0,1] \times R \times R \rightarrow R$ be continuous. Assume that $f$ meets the following condition: there exist $l_{1}>0, l_{2}>0$ such that

$$
\begin{gathered}
\left|\phi_{q}\left(f\left(t, u, u^{\prime}\right)\right)-\phi_{q}\left(f\left(t, v, v^{\prime}\right)\right)\right| \leq l_{1}|u-v|+l_{2}\left|u^{\prime}-v^{\prime}\right| \\
\left(\frac{T^{\alpha-1}}{\Gamma(\alpha)}+\frac{T^{\alpha}}{\Gamma(\alpha+1)}+\frac{a T^{\alpha} \Gamma(2-\beta)}{|1-a| \Gamma(\alpha-\beta+1)}\right. \\
+\frac{a T^{\alpha}}{|1-a| \Gamma(\alpha+1)}+\frac{T^{\alpha-1} \Gamma(2-\beta)}{\Gamma(\alpha-\beta+1)} \\
\left.+\frac{T^{\alpha} \Gamma(2-\beta)}{\Gamma(\alpha-\beta+1)}\right)\left(l_{1}+l_{2}\right)<1 .
\end{gathered}
$$

Then the problem (5) has a unique solution on $[0,1]$ for any $\lambda \geq 0$.

Proof. From (18) and (19), we have, for $u_{1}, u_{2} \in X$,

$$
\begin{aligned}
& \left|\mathrm{F} u_{2}(t)-\mathrm{F} u_{1}(t)\right| \\
& \leq \frac{1}{\Gamma(\alpha)} \int_{0}^{t}(t-s)^{\alpha-1} \mid \phi_{q}\left(f\left(s, u_{2}(s), u_{2}^{\prime}(s)\right)\right) \\
& -\phi_{q}\left(f\left(s, u_{1}(s), u_{1}^{\prime}(s)\right)\right) \mid d s
\end{aligned}
$$


6

Discrete Dynamics in Nature and Society

$$
\begin{aligned}
& +\frac{a}{\left|(-1)^{\theta}+a\right| \Gamma(\alpha)} \\
& +\frac{a \Gamma(2-\beta)}{\left|(-1)^{\theta}+a\right| T^{-\beta} \Gamma(\alpha-\beta)} \\
& \times \int_{0}^{T}(T-s)^{\alpha-1} \mid \phi_{q}\left(f\left(s, u_{2}(s), u_{2}^{\prime}(s)\right)\right) \\
& \times \int_{0}^{T}(T-s)^{\alpha-\beta-1} d s \\
& -\phi_{q}\left(f\left(s, u_{1}(s), u_{1}^{\prime}(s)\right)\right) \mid d s \\
& +\frac{a \Gamma(2-\beta)}{\left|(-1)^{\theta}+a\right| T^{-\beta} \Gamma(\alpha-\beta)} \\
& +\frac{a}{\left|(-1)^{\theta}+a\right| \Gamma(\alpha)} \int_{0}^{T}(T-s)^{\alpha-1} d s \\
& \left.+t\left[\frac{\Gamma(2-\beta)}{T^{1-\beta} \Gamma(\alpha-\beta)} \int_{0}^{T}(T-s)^{\alpha-\beta-1} d s\right]\right) \\
& \times \int_{0}^{T}(T-s)^{\alpha-\beta-1} \mid \phi_{q}\left(f\left(s, u_{2}(s), u_{2}^{\prime}(s)\right)\right) \\
& -\phi_{q}\left(f\left(s, u_{1}(s), u_{1}^{\prime}(s)\right)\right) \mid d s \\
& +t\left[\frac{\Gamma(2-\beta)}{T^{1-\beta} \Gamma(\alpha-\beta)}\right. \\
& \times\left(l_{1}+l_{2}\right)\left\|u_{2}-u_{1}\right\|_{1} \\
& \leq\left(\frac{T^{\alpha}}{\Gamma(\alpha+1)}+\frac{a T^{\alpha} \Gamma(2-\beta)}{|1-a| \Gamma(\alpha-\beta+1)}\right. \\
& \left.+\frac{a T^{\alpha}}{|1-a| \Gamma(\alpha+1)}+\frac{T^{\alpha} \Gamma(2-\beta)}{\Gamma(\alpha-\beta+1)}\right) \\
& \times \int_{0}^{T}(T-s)^{\alpha-\beta-1} \mid \phi_{q}\left(f\left(s, u_{2}(s), u_{2}^{\prime}(s)\right)\right) \\
& \times\left(l_{1}+l_{2}\right)\left\|u_{2}-u_{1}\right\|_{1}, \\
& \left.-\phi_{q}\left(f\left(s, u_{1}(s), u_{1}^{\prime}(s)\right)\right) \mid d s\right] \\
& \left|\mathrm{F}^{\prime} u_{2}(t)-\mathrm{F}^{\prime} u_{1}(t)\right| \\
& \leq \frac{1}{\Gamma(\alpha)} \int_{0}^{t}(t-s)^{\alpha-1}\left[l_{1}\left|u_{2}(s)-u_{1}(s)\right|\right. \\
& \leq \frac{1}{\Gamma(\alpha-1)} \int_{0}^{t}(t-s)^{\alpha-2} \mid \phi_{q}\left(f\left(s, u_{2}(s), u_{2}^{\prime}(s)\right)\right) \\
& \left.+l_{2}\left|u_{2}^{\prime}(s)-u_{1}^{\prime}(s)\right|\right] d s \\
& -\phi_{q}\left(f\left(s, u_{1}(s), u_{1}^{\prime}(s)\right)\right) \mid d s \\
& +\frac{\Gamma(2-\beta)}{T^{1-\beta} \Gamma(\alpha-\beta)} \\
& \times \int_{0}^{T}(T-s)^{\alpha-\beta-1} \mid \phi_{q}\left(f\left(s, u_{2}(s), u_{2}^{\prime}(s)\right)\right) \\
& -\phi_{q}\left(f\left(s, u_{1}(s), u_{1}^{\prime}(s)\right)\right) \mid d s \\
& \left.+l_{2}\left|u_{2}^{\prime}(s)-u_{1}^{\prime}(s)\right|\right] d s \\
& +\frac{a \Gamma(2-\beta)}{\left|(-1)^{\theta}+a\right| T^{-\beta} \Gamma(\alpha-\beta)} \\
& \times \int_{0}^{T}(T-s)^{\alpha-\beta-1}\left[l_{1}\left|u_{2}(s)-u_{1}(s)\right|\right. \\
& \left.+l_{2}\left|u_{2}^{\prime}(s)-u_{1}^{\prime}(s)\right|\right] d s \\
& +t\left[\frac{\Gamma(2-\beta)}{T^{1-\beta} \Gamma(\alpha-\beta)}\right. \\
& \leq\left(\frac{1}{\Gamma(\alpha-1)} \int_{0}^{t}(t-s)^{\alpha-2} d s\right. \\
& \times \int_{0}^{T}(T-s)^{\alpha-\beta-1}\left[l_{1}\left|u_{2}(s)-u_{1}(s)\right|\right. \\
& \left.+\frac{\Gamma(2-\beta)}{T^{1-\beta} \Gamma(\alpha-\beta)} \int_{0}^{T}(T-s)^{\alpha-\beta-1} d s\right) \\
& \times\left(l_{1}+l_{2}\right)\left\|u_{2}-u_{1}\right\|_{1} \\
& \left.\left.+l_{2}\left|u_{2}^{\prime}(s)-u_{1}^{\prime}(s)\right|\right] d s\right] \\
& \leq\left(\frac{1}{\Gamma(\alpha)} \int_{0}^{t}(t-s)^{\alpha-1} d s\right. \\
& \leq\left(\frac{T^{\alpha-1}}{\Gamma(\alpha)}+\frac{T^{\alpha-1} \Gamma(2-\beta)}{\Gamma(\alpha-\beta+1)}\right)\left(l_{1}+l_{2}\right)\left\|u_{2}-u_{1}\right\|_{1} .
\end{aligned}
$$


Thus,

$$
\begin{aligned}
\left\|\mathrm{F} u_{2}-\mathrm{F} u_{1}\right\|_{1} \leq( & \frac{T^{\alpha-1}}{\Gamma(\alpha)}+\frac{T^{\alpha}}{\Gamma(\alpha+1)}+\frac{a T^{\alpha} \Gamma(2-\beta)}{|1-a| \Gamma(\alpha-\beta+1)} \\
& +\frac{a T^{\alpha}}{|1-a| \Gamma(\alpha+1)}+\frac{T^{\alpha-1} \Gamma(2-\beta)}{\Gamma(\alpha-\beta+1)} \\
& \left.+\frac{T^{\alpha} \Gamma(2-\beta)}{\Gamma(\alpha-\beta+1)}\right)\left(l_{1}+l_{2}\right)\left\|u_{2}-u_{1}\right\|_{1} .
\end{aligned}
$$

It follows from (29) that $F$ is a contraction. Thus, the conclusion of the theorem follows from the contraction mapping principle.

Theorem 7. Let $1<p<2$. Assume that $f$ meets the following condition: there exist $d_{1}>0, d_{2}>0, d>0$ such that

$$
\begin{gathered}
\left|f\left(t, u, u^{\prime}\right)\right| \leq M, \\
\left|f\left(t, u, u^{\prime}\right)-f\left(t, v, v^{\prime}\right)\right| \leq d_{1}|u-v|+d_{2}\left|u^{\prime}-v^{\prime}\right|, \\
\left(\frac{T^{\alpha-1}}{\Gamma(\alpha)}+\frac{T^{\alpha}}{\Gamma(\alpha+1)}+\frac{a T^{\alpha} \Gamma(2-\beta)}{|1-a| \Gamma(\alpha-\beta+1)}\right. \\
+\frac{a T^{\alpha}}{|1-a| \Gamma(\alpha+1)}+\frac{T^{\alpha-1} \Gamma(2-\beta)}{\Gamma(\alpha-\beta+1)} \\
\left.+\frac{T^{\alpha} \Gamma(2-\beta)}{\Gamma(\alpha-\beta+1)}\right)(q-1) M^{(q-2)}\left(d_{1}+d_{2}\right)<1 .
\end{gathered}
$$

Then the problem (5) has unique solution on $[0,1]$ for

$$
\begin{gathered}
0 \leq \lambda \leq\left(\frac{a \Gamma(2-\beta)}{|1-a| b T^{-\beta}}+\frac{\Gamma(2-\beta)}{b T^{-\beta}}+\frac{\Gamma(2-\beta)}{b T^{1-\beta}}\right)^{-1} \\
\times\left[d-\left(\frac{T^{\alpha-1}}{\Gamma(\alpha)}+\frac{T^{\alpha-1} \Gamma(2-\beta)}{\Gamma(\alpha-\beta+1)}\right.\right. \\
+\frac{T^{\alpha}}{\Gamma(\alpha+1)}+\frac{a T^{\alpha}}{|1-a| \Gamma(\alpha+1)} \\
+\frac{a T^{\alpha} \Gamma(2-\beta)}{|1-a| \Gamma(\alpha-\beta+1)} \\
\left.\left.+\frac{T^{\alpha} \Gamma(2-\beta)}{\Gamma(\alpha-\beta+1)}\right) \phi_{q}(M)\right]
\end{gathered}
$$

Proof. Let

$$
B_{d}=\left\{u \mid\|u\|_{1} \leq d, u \in X\right\},
$$

where

$$
\begin{gathered}
d \geq\left(\frac{T^{\alpha-1}}{\Gamma(\alpha)}+\frac{T^{\alpha-1} \Gamma(2-\beta)}{\Gamma(\alpha-\beta+1)}+\frac{T^{\alpha}}{\Gamma(\alpha+1)}+\frac{a T^{\alpha}}{|1-a| \Gamma(\alpha+1)}\right. \\
\left.+\frac{a T^{\alpha} \Gamma(2-\beta)}{|1-a| \Gamma(\alpha-\beta+1)}+\frac{T^{\alpha} \Gamma(2-\beta)}{\Gamma(\alpha-\beta+1)}\right) \phi_{q}(M) \\
+\frac{a \Gamma(2-\beta)}{|1-a| b T^{-\beta}} \lambda+\frac{\Gamma(2-\beta)}{b T^{-\beta}} \lambda+\frac{\Gamma(2-\beta)}{b T^{1-\beta}} \lambda .
\end{gathered}
$$

By (18) and (19), we have, for $u \in B_{d}$,

$|\mathrm{F} u(t)| \leq \frac{1}{\Gamma(\alpha)} \int_{0}^{t}(t-s)^{\alpha-1}\left|\phi_{q}\left(f\left(s, u(s), u^{\prime}(s)\right)\right)\right| d s$

$$
\begin{aligned}
& +\frac{a}{\left|(-1)^{\theta}+a\right| \Gamma(\alpha)} \\
& \times \int_{0}^{T}(T-s)^{\alpha-1}\left|\phi_{q}\left(f\left(s, u(s), u^{\prime}(s)\right)\right)\right| d s \\
& +\frac{a \Gamma(2-\beta)}{\left|(-1)^{\theta}+a\right| T^{-\beta} \Gamma(\alpha-\beta)} \\
& \times \int_{0}^{T}(T-s)^{\alpha-\beta-1}\left|\phi_{q}\left(f\left(s, u(s), u^{\prime}(s)\right)\right)\right| d s \\
& +\left|\frac{a \Gamma(2-\beta)}{\left[1+(-1)^{\theta} a\right] b T^{-\beta}} \lambda\right| \\
& +t\left[\frac{\Gamma(2-\beta)}{T^{1-\beta} \Gamma(\alpha-\beta)}\right.
\end{aligned}
$$$$
\times \int_{0}^{T}(T-s)^{\alpha-\beta-1}\left|\phi_{q}\left(f\left(s, u(s), u^{\prime}(s)\right)\right)\right| d s
$$$$
\left.+\left|\frac{(-1)^{\theta} \Gamma(2-\beta)}{b T^{1-\beta}} \lambda\right|\right]
$$$$
\leq \frac{1}{\Gamma(\alpha)} \int_{0}^{t}(t-s)^{\alpha-1} \phi_{q}(M) d s
$$$$
+\frac{a}{|1-a| \Gamma(\alpha)} \int_{0}^{T}(T-s)^{\alpha-1} \phi_{q}(M) d s
$$$$
+\frac{a \Gamma(2-\beta)}{|1-a| T^{-\beta} \Gamma(\alpha-\beta)} \int_{0}^{T}(T-s)^{\alpha-\beta-1} \phi_{q}(M) d s
$$$$
+\frac{a \Gamma(2-\beta)}{|1-a| b T^{-\beta}} \lambda
$$

$$
+t\left[\frac{\Gamma(2-\beta)}{T^{1-\beta} \Gamma(\alpha-\beta)}\right.
$$

$$
\left.\times \int_{0}^{T}(T-s)^{\alpha-\beta-1} \phi_{q}(M) d s+\frac{\Gamma(2-\beta)}{b T^{1-\beta}} \lambda\right]
$$




$$
\begin{aligned}
& \leq\left(\frac{T^{\alpha}}{\Gamma(\alpha+1)}+\frac{a T^{\alpha}}{|1-a| \Gamma(\alpha+1)}\right. \\
& \left.\quad+\frac{a T^{\alpha} \Gamma(2-\beta)}{|1-a| \Gamma(\alpha-\beta+1)}+\frac{T^{\alpha} \Gamma(2-\beta)}{\Gamma(\alpha-\beta+1)}\right) \phi_{q}(M) \\
& +\frac{a \Gamma(2-\beta)}{|1-a| b T^{-\beta}} \lambda+\frac{\Gamma(2-\beta)}{b T^{-\beta}} \lambda,
\end{aligned}
$$

This, together with (36), yields that

$$
\|\mathrm{F} u(t)\|_{1} \leq d
$$

Hence,

$$
\mathrm{F}: B_{d} \longrightarrow B_{d}
$$

In view of $1<p<2$, we have $q>2$. Thus, by the following property of p-Laplacian operator:

if $p>2,|x|,|y| \leq c$, then $\left|\varphi_{p}(x)-\varphi_{p}(y)\right| \leq(p-1) c^{(p-2)} \mid x-$ $y \mid$; we have, for $u \in B_{d}$,

$$
\begin{aligned}
& \left|\mathrm{F} u_{2}(t)-\mathrm{F} u_{1}(t)\right| \\
& \leq \frac{1}{\Gamma(\alpha)} \int_{0}^{t}(t-s)^{\alpha-1} \mid \phi_{q}\left(f\left(s, u_{2}(s), u_{2}^{\prime}(s)\right)\right) \\
& -\phi_{q}\left(f\left(s, u_{1}(s), u_{1}^{\prime}(s)\right)\right) \mid d s \\
& +\frac{a}{\left|(-1)^{\theta}+a\right| \Gamma(\alpha)} \\
& \times \int_{0}^{T}(T-s)^{\alpha-1} \mid \phi_{q}\left(f\left(s, u_{2}(s), u_{2}^{\prime}(s)\right)\right) \\
& -\phi_{q}\left(f\left(s, u_{1}(s), u_{1}^{\prime}(s)\right)\right) \mid d s \\
& +\frac{a \Gamma(2-\beta)}{\left|(-1)^{\theta}+a\right| T^{-\beta} \Gamma(\alpha-\beta)} \\
& \times \int_{0}^{T}(T-s)^{\alpha-\beta-1} \mid \phi_{q}\left(f\left(s, u_{2}(s), u_{2}^{\prime}(s)\right)\right) \\
& -\phi_{q}\left(f\left(s, u_{1}(s), u_{1}^{\prime}(s)\right)\right) \mid d s \\
& +t\left[\frac{\Gamma(2-\beta)}{T^{1-\beta} \Gamma(\alpha-\beta)}\right. \\
& \times \int_{0}^{T}(T-s)^{\alpha-\beta-1} \mid \phi_{q}\left(f\left(s, u_{2}(s), u_{2}^{\prime}(s)\right)\right) \\
& \left.-\phi_{q}\left(f\left(s, u_{1}(s), u_{1}^{\prime}(s)\right)\right) \mid d s\right]
\end{aligned}
$$

$$
\begin{aligned}
& \leq \frac{(q-1) M^{(q-2)}}{\Gamma(\alpha)} \\
& \quad \times \int_{0}^{t}(t-s)^{\alpha-1} \mid f\left(s, u_{2}(s), u_{2}^{\prime}(s)\right) \\
& +\frac{a(q-1) M^{(q-2)}}{\left|(-1)^{\theta}+a\right| \Gamma(\alpha)} \\
& \left.\quad \times \int_{0}^{T}(T-s)^{\alpha-1} \mid f\left(s, u_{2}(s), u_{2}^{\prime}(s)\right), u_{1}^{\prime}(s)\right) \mid d s \\
& \quad-f\left(s, u_{1}(s), u_{1}^{\prime}(s)\right) \mid d s
\end{aligned}
$$

$$
\begin{aligned}
& +\frac{a(q-1) M^{(q-2)} \Gamma(2-\beta)}{\left|(-1)^{\theta}+a\right| T^{-\beta} \Gamma(\alpha-\beta)} \\
& \times \int_{0}^{T}(T-s)^{\alpha-\beta-1} \mid f\left(s, u_{2}(s), u_{2}^{\prime}(s)\right) \\
& -f\left(s, u_{1}(s), u_{1}^{\prime}(s)\right) \mid d s
\end{aligned}
$$$$
+t\left[\frac{(q-1) M^{(q-2)} \Gamma(2-\beta)}{T^{1-\beta} \Gamma(\alpha-\beta)}\right.
$$$$
\times \int_{0}^{T}(T-s)^{\alpha-\beta-1} \mid f\left(s, u_{2}(s), u_{2}^{\prime}(s)\right)
$$$$
\left.-f\left(s, u_{1}(s), u_{1}^{\prime}(s)\right) \mid d s\right]
$$$$
\leq \frac{(q-1) M^{(q-2)}}{\Gamma(\alpha)} \int_{0}^{t}(t-s)^{\alpha-1}\left[d_{1}\left|u_{2}(s)-u_{1}(s)\right|\right.
$$$$
\left.+d_{2}\left|u_{2}^{\prime}(s)-u_{1}^{\prime}(s)\right|\right] d s
$$$$
\begin{aligned}
& +\frac{a(q-1) M^{(q-2)}}{\left|(-1)^{\theta}+a\right| \Gamma(\alpha)} \\
& \times \int_{0}^{T}(T-s)^{\alpha-1}\left[d_{1}\left|u_{2}(s)-u_{1}(s)\right|\right. \\
& \left.+d_{2}\left|u_{2}^{\prime}(s)-u_{1}^{\prime}(s)\right|\right] d s
\end{aligned}
$$$$
+\frac{a(q-1) M^{(q-2)} \Gamma(2-\beta)}{\left|(-1)^{\theta}+a\right| T^{-\beta} \Gamma(\alpha-\beta)}
$$$$
\times \int_{0}^{T}(T-s)^{\alpha-\beta-1}\left[d_{1}\left|u_{2}(s)-u_{1}(s)\right|\right.
$$$$
\left.+d_{2}\left|u_{2}^{\prime}(s)-u_{1}^{\prime}(s)\right|\right] d s
$$ 


$$
\begin{aligned}
& +t\left[\frac{(q-1) M^{(q-2)} \Gamma(2-\beta)}{T^{1-\beta} \Gamma(\alpha-\beta)}\right. \\
& \times \int_{0}^{T}(T-s)^{\alpha-\beta-1}\left[d_{1}\left|u_{2}(s)-u_{1}(s)\right|\right. \\
& \left.\left.+d_{2}\left|u_{2}^{\prime}(s)-u_{1}^{\prime}(s)\right|\right] d s\right] \\
& \leq\left(\frac{1}{\Gamma(\alpha)} \int_{0}^{t}(t-s)^{\alpha-1} d s\right. \\
& +\frac{a \Gamma(2-\beta)}{\left|(-1)^{\theta}+a\right| T^{-\beta} \Gamma(\alpha-\beta)} \int_{0}^{T}(T-s)^{\alpha-\beta-1} d s \\
& +\frac{a}{\left|(-1)^{\theta}+\alpha\right| \Gamma(\alpha)} \int_{0}^{T}(T-s)^{\alpha-1} d s \\
& \left.+t\left[\frac{\Gamma(2-\beta)}{T^{1-\beta} \Gamma(\alpha-\beta)} \int_{0}^{T}(T-s)^{\alpha-\beta-1} d s\right]\right) \\
& \times(q-1) M^{(q-2)}\left(d_{1}+d_{2}\right)\left\|u_{2}-u_{1}\right\|_{1} \\
& \leq\left(\frac{T^{\alpha}}{\Gamma(\alpha+1)}+\frac{a T^{\alpha} \Gamma(2-\beta)}{|1-a| \Gamma(\alpha-\beta+1)}\right. \\
& \left.+\frac{a T^{\alpha}}{|1-a| \Gamma(\alpha+1)}+\frac{T^{\alpha} \Gamma(2-\beta)}{\Gamma(\alpha-\beta+1)}\right) \\
& \times(q-1) M^{(q-2)}\left(d_{1}+d_{2}\right)\left\|u_{2}-u_{1}\right\|_{1}, \\
& \left|\mathrm{~F}^{\prime} u_{2}(t)-\mathrm{F}^{\prime} u_{1}(t)\right| \\
& \leq \frac{1}{\Gamma(\alpha-1)} \\
& \times \int_{0}^{t}(t-s)^{\alpha-2} \mid \phi_{q}\left(f\left(s, u_{2}(s), u_{2}^{\prime}(s)\right)\right) \\
& -\phi_{q}\left(f\left(s, u_{1}(s), u_{1}^{\prime}(s)\right)\right) \mid d s \\
& +\frac{\Gamma(2-\beta)}{T^{1-\beta} \Gamma(\alpha-\beta)} \\
& \times \int_{0}^{T}(T-s)^{\alpha-\beta-1} \mid \phi_{q}\left(f\left(s, u_{2}(s), u_{2}^{\prime}(s)\right)\right) \\
& -\phi_{q}\left(f\left(s, u_{1}(s), u_{1}^{\prime}(s)\right)\right) \mid d s \\
& \leq \frac{(q-1) M^{(q-2)}}{\Gamma(\alpha-1)} \int_{0}^{t}(t-s)^{\alpha-2} \mid f\left(s, u_{2}(s), u_{2}^{\prime}(s)\right) \\
& -f\left(s, u_{1}(s), u_{1}^{\prime}(s)\right) \mid d s \\
& +\frac{(q-1) M^{(q-2)} \Gamma(2-\beta)}{T^{1-\beta} \Gamma(\alpha-\beta)}
\end{aligned}
$$

$$
\begin{aligned}
\times \int_{0}^{T}(T-s)^{\alpha-\beta-1} \mid & f\left(s, u_{2}(s), u_{2}^{\prime}(s)\right) \\
& -f\left(s, u_{1}(s), u_{1}^{\prime}(s)\right) \mid d s
\end{aligned}
$$$$
\leq \frac{(q-1) M^{(q-2)}}{\Gamma(\alpha-1)} \int_{0}^{t}(t-s)^{\alpha-2}\left[d_{1}\left|u_{2}(s)-u_{1}(s)\right|\right.
$$$$
\left.+d_{2}\left|u_{2}^{\prime}(s)-u_{1}^{\prime}(s)\right|\right] d s
$$$$
+\frac{(q-1) M^{(q-2)} \Gamma(2-\beta)}{T^{1-\beta} \Gamma(\alpha-\beta)}
$$$$
\times \int_{0}^{T}(T-s)^{\alpha-\beta-1}\left[d_{1}\left|u_{2}(s)-u_{1}(s)\right|\right.
$$$$
\left.+d_{2}\left|u_{2}^{\prime}(s)-u_{1}^{\prime}(s)\right|\right] d s
$$$$
\leq\left(\frac{1}{\Gamma(\alpha-1)} \int_{0}^{t}(t-s)^{\alpha-2} d s\right.
$$$$
\left.+\frac{\Gamma(2-\beta)}{T^{1-\beta} \Gamma(\alpha-\beta)} \int_{0}^{T}(T-s)^{\alpha-\beta-1} d s\right)
$$$$
\times(q-1) M^{(q-2)}\left(d_{1}+d_{2}\right)\left\|u_{2}-u_{1}\right\|_{1}
$$$$
\leq\left(\frac{T^{\alpha-1}}{\Gamma(\alpha)}+\frac{T^{\alpha-1} \Gamma(2-\beta)}{\Gamma(\alpha-\beta+1)}\right)
$$$$
\times(q-1) M^{(q-2)}\left(d_{1}+d_{2}\right)\left\|u_{2}-u_{1}\right\|_{1} .
$$

Thus,

$$
\begin{aligned}
\| \mathrm{F} u_{2}- & \mathrm{F} u_{1} \|_{1} \\
\leq & \frac{T^{\alpha-1}}{\Gamma(\alpha)}+\frac{T^{\alpha}}{\Gamma(\alpha+1)}+\frac{a T^{\alpha} \Gamma(2-\beta)}{|1-a| \Gamma(\alpha-\beta+1)} \\
& \left.+\frac{a T^{\alpha}}{|1-a| \Gamma(\alpha+1)}+\frac{T^{\alpha-1} \Gamma(2-\beta)}{\Gamma(\alpha-\beta+1)}+\frac{T^{\alpha} \Gamma(2-\beta)}{\Gamma(\alpha-\beta+1)}\right) \\
& \times(q-1) M^{(q-2)}\left(d_{1}+d_{2}\right)\left\|u_{2}-u_{1}\right\|_{1} .
\end{aligned}
$$

It follows from (33) that $\mathrm{F}$ is a contraction. Thus, the conclusion of the theorem follows by the contraction mapping principle.

\section{Examples}

Example 8. Consider the following boundary value problem:

$$
\phi_{2}\left({ }^{c} D_{0+}^{3 / 2} u(t)\right)=f\left(t, u(t), u^{\prime}(t)\right), \quad 0<t<1,
$$




$$
\begin{gathered}
u(0)+(-1)^{\theta} 2 u(1)=0, \\
{ }^{c} D_{0+}^{1 / 2} u(0)+(-1)^{\theta} \frac{3 \sqrt{\pi}}{2}{ }^{c} D_{0+}^{1 / 2} u(1)=\lambda, \quad \theta=0,1,
\end{gathered}
$$

where

$$
\begin{aligned}
& T=1, \quad \alpha=\frac{3}{2}, \quad \beta=\frac{1}{2}, \\
& a=2, \quad b=\frac{3 \sqrt{\pi}}{2} .
\end{aligned}
$$

Let

$$
\begin{gathered}
d=2, \quad p=2, \\
f\left(t, u(t), u^{\prime}(t)\right)=\frac{t}{100} \frac{u}{5+|u|+\left|u^{\prime}\right|} .
\end{gathered}
$$

By computation, we deduce that

$$
\begin{aligned}
0 \leq \lambda \leq & \frac{d}{2}\left(\frac{a \Gamma(2-\beta)}{|1-a| b T^{-\beta}}+\frac{\Gamma(2-\beta)}{b T^{-\beta}}+\frac{\Gamma(2-\beta)}{b T^{1-\beta}}\right)^{-1} \\
= & \left(\frac{2 \Gamma(3 / 2)}{(3 \sqrt{\pi} / 2)}+\frac{\Gamma(3 / 2)}{(3 \sqrt{\pi} / 2)}+\frac{\Gamma(3 / 2)}{(3 \sqrt{\pi} / 2)}\right)^{-1} \\
= & \left(\frac{2(\sqrt{\pi} / 2)}{(3 \sqrt{\pi} / 2)}+\frac{\sqrt{\pi} / 2}{(3 \sqrt{\pi} / 2)}+\frac{\sqrt{\pi} / 2}{(3 \sqrt{\pi} / 2)}\right)^{-1}=\frac{3}{4}, \\
\phi_{2}(\gamma)=\gamma \leq & \frac{1}{2}\left(\frac{T^{\alpha-1}}{\Gamma(\alpha)}+\frac{T^{\alpha}}{\Gamma(\alpha+1)}\right. \\
& +\frac{a T^{\alpha}}{|1-a| \Gamma(\alpha+1)}+\frac{a T^{\alpha} \Gamma(2-\beta)}{|1-a| \Gamma(\alpha-\beta+1)} \\
& \left.+\frac{T^{\alpha} \Gamma(2-\beta)}{\Gamma(\alpha-\beta+1)}+\frac{T^{\alpha-1} \Gamma(2-\beta)}{\Gamma(\alpha-\beta+1)}\right)^{-1} \\
= & \frac{1}{2}\left(\frac{1}{\Gamma(3 / 2)}+\frac{1}{\Gamma(5 / 2)}+\frac{2}{\Gamma(5 / 2)}+\frac{2 \Gamma(3 / 2)}{\Gamma(2)}\right. \\
& \left.+\frac{\Gamma(3 / 2)}{\Gamma(2)}+\frac{\Gamma(3 / 2)}{\Gamma(2)}\right)^{-1} \\
= & \frac{1}{2}\left(\frac{18+6 \pi}{3 \sqrt{\pi}}\right)^{-1}=\frac{3 \sqrt{\pi}}{36+12 \pi}<\frac{1}{5} .
\end{aligned}
$$

Thus, let $\gamma=1 / 9$; we have

$$
f\left(t, u(t), u^{\prime}(t)\right)=\frac{t}{100} \frac{u}{5+|u|+\left|u^{\prime}\right|}<\gamma \phi_{2}(|u|)=\frac{1}{9}|u| .
$$

Hence, by Theorem 5, BVP (42) has at least one solution for $0 \leq \lambda \leq 3 / 4$.
Example 9. Consider the following boundary value problem:

$$
\begin{gathered}
\phi_{2}\left({ }^{c} D_{0+}^{3 / 2} u(t)\right)=f\left(t, u(t), u^{\prime}(t)\right), \quad 0<t<1, \\
u(0)+(-1)^{\theta} 2 u(1)=0, \\
{ }^{c} D_{0+}^{1 / 2} u(0)+(-1)^{\theta} \sqrt{\pi}{ }^{c} D_{0+}^{1 / 2} u(1)=\lambda, \quad \theta=0,1,
\end{gathered}
$$

where

$$
\begin{aligned}
& T=1, \quad \alpha=\frac{3}{2}, \quad \beta=\frac{1}{2}, \\
& a=2, \quad b=\sqrt{\pi}, \quad \lambda \geq 0 .
\end{aligned}
$$

Let

$$
p=2 \text {, }
$$

$f\left(t, u(t), u^{\prime}(t)\right)$

$$
=\frac{1}{(t+\cos t+10)^{2}}\left(\frac{\left|u(t)+u^{\prime}(t)\right|}{1+\left|u(t)+u^{\prime}(t)\right|}+10(1+\sin t)\right) .
$$

By computation, we deduce that

$$
\begin{aligned}
& \left|f\left(t, u(t), u^{\prime}(t)\right)-f\left(t, v(t), v^{\prime}(t)\right)\right| \\
& \quad \leq \frac{1}{100}\left(|u(t)-v(t)|+\left|u^{\prime}(t)-v^{\prime}(t)\right|\right) .
\end{aligned}
$$

Let

$$
l_{1}=l_{2}=\frac{1}{100} .
$$

Thus,

$$
\begin{aligned}
& \left(\frac{T^{\alpha-1}}{\Gamma(\alpha)}+\frac{T^{\alpha}}{\Gamma(\alpha+1)}+\frac{a T^{\alpha} \Gamma(2-\beta)}{|1-a| \Gamma(\alpha-\beta+1)}\right. \\
& +\frac{a T^{\alpha}}{|1-a| \Gamma(\alpha+1)}+\frac{T^{\alpha-1} \Gamma(2-\beta)}{\Gamma(\alpha-\beta+1)} \\
& \left.+\frac{T^{\alpha} \Gamma(2-\beta)}{\Gamma(\alpha-\beta+1)}\right)\left(l_{1}+l_{2}\right) \\
& =\left(\frac{1}{\Gamma(3 / 2)}+\frac{1}{\Gamma(5 / 2)}+\frac{2 \Gamma(3 / 2)}{\Gamma(2)}+\frac{2}{\Gamma(5 / 2)}\right. \\
& \left.\quad+\frac{\Gamma(3 / 2)}{\Gamma(2)}+\frac{\Gamma(3 / 2)}{\Gamma(2)}\right)\left(\frac{1}{100}+\frac{1}{100}\right) \\
& =\left(\frac{1}{(\sqrt{\pi} / 2)}+\frac{1}{(3 \sqrt{\pi} / 4)}+\frac{2(\sqrt{\pi} / 2)}{1}+\frac{2}{(3 \sqrt{\pi} / 4)}\right. \\
& \left.\quad+\frac{(\sqrt{\pi} / 2)}{1}+\frac{(\sqrt{\pi} / 2)}{1}\right)\left(\frac{1}{100}+\frac{1}{100}\right) \\
& =\frac{3+\pi}{25 \sqrt{\pi}}<1 .
\end{aligned}
$$

Hence, by Theorem 6, BVP (47) has a unique solution. 
Example 10. Consider the following boundary value problem:

$$
\begin{gathered}
\phi_{4 / 3}\left({ }^{c} D_{0+}^{3 / 2} u(t)\right)=f\left(t, u(t), u^{\prime}(t)\right), 0<t<1, \\
u(0)+(-1)^{\theta} 2 u(1)=0, \\
{ }^{c} D_{0+}^{1 / 2} u(0)+(-1)^{\theta} \sqrt{\pi}{ }^{c} D_{0+}^{1 / 2} u(1)=\lambda, \theta=0,1,
\end{gathered}
$$

where

$$
\begin{array}{lll}
p=\frac{4}{3}, & T=1, & \alpha=\frac{3}{2}, \\
\beta=\frac{1}{2}, & a=2, & b=\sqrt{\pi} .
\end{array}
$$

Let

$$
\begin{aligned}
f & \left(t, u(t), u^{\prime}(t)\right) \\
& =\frac{1}{(t+\cos t+10)^{2}}\left(\frac{\left|u(t)+u^{\prime}(t)\right|}{1+\left|u(t)+u^{\prime}(t)\right|}+10(1+\sin t)\right) .
\end{aligned}
$$

Thus,

$$
\begin{gathered}
q=4, \\
\left|f\left(t, u(t), u^{\prime}(t)\right)\right| \leq \frac{1}{100}(1+12)=\frac{13}{100}=M \\
(q-1) M^{(q-2)}=(4-1) M^{4-2}=3\left(\frac{13}{100}\right)^{2}<1 .
\end{gathered}
$$

By Example 9, we know that

$$
\begin{aligned}
& \left|f\left(t, u(t), u^{\prime}(t)\right)-f\left(t, v(t), v^{\prime}(t)\right)\right| \\
& \quad \leq \frac{1}{100}\left(|u(t)-v(t)|+\left|u^{\prime}(t)-v^{\prime}(t)\right|\right) .
\end{aligned}
$$

Let

$$
d_{1}=d_{2}=\frac{1}{100}
$$

It follows from Example 9 that

$$
\begin{aligned}
& \left(\frac{T^{\alpha-1}}{\Gamma(\alpha)}+\frac{T^{\alpha}}{\Gamma(\alpha+1)}+\frac{a T^{\alpha} \Gamma(2-\beta)}{|1-a| \Gamma(\alpha-\beta+1)}\right. \\
& +\frac{a T^{\alpha}}{|1-a| \Gamma(\alpha+1)}+\frac{T^{\alpha-1} \Gamma(2-\beta)}{\Gamma(\alpha-\beta+1)} \\
& \left.+\frac{T^{\alpha} \Gamma(2-\beta)}{\Gamma(\alpha-\beta+1)}\right)\left(d_{1}+d_{2}\right)<1 .
\end{aligned}
$$

On the other hand, we have

$$
\begin{aligned}
& \left(\frac{a \Gamma(2-\beta)}{|1-a| b T^{-\beta}}+\frac{\Gamma(2-\beta)}{b T^{-\beta}}+\frac{\Gamma(2-\beta)}{b T^{1-\beta}}\right)^{-1} \\
& \quad=\left(\frac{2 \Gamma(3 / 2)}{\sqrt{\pi}}+\frac{\Gamma(3 / 2)}{\sqrt{\pi}}+\frac{\Gamma(3 / 2)}{\sqrt{\pi}}\right)^{-1}=\frac{1}{2} .
\end{aligned}
$$

Let

$$
d=2 \times \frac{3+\pi}{25 \sqrt{\pi}} .
$$

Thus,

$$
\begin{aligned}
& \left(\frac{T^{\alpha-1}}{\Gamma(\alpha)}+\frac{T^{\alpha}}{\Gamma(\alpha+1)}+\frac{a T^{\alpha} \Gamma(2-\beta)}{|1-a| \Gamma(\alpha-\beta+1)}\right. \\
& \quad+\frac{a T^{\alpha}}{|1-a| \Gamma(\alpha+1)}+\frac{T^{\alpha-1} \Gamma(2-\beta)}{\Gamma(\alpha-\beta+1)} \\
& \left.\quad+\frac{T^{\alpha} \Gamma(2-\beta)}{\Gamma(\alpha-\beta+1)}\right)(q-1) M^{(q-2)}\left(d_{1}+d_{2}\right)<1,
\end{aligned}
$$

$0 \leq \lambda$

$$
\begin{gathered}
\leq\left(\frac{a \Gamma(2-\beta)}{|1-a| b T^{-\beta}}+\frac{\Gamma(2-\beta)}{b T^{-\beta}}+\frac{\Gamma(2-\beta)}{b T^{1-\beta}}\right)^{-1} \\
\times\left[d-\left(\frac{T^{\alpha-1}}{\Gamma(\alpha)}+\frac{T^{\alpha-1} \Gamma(2-\beta)}{\Gamma(\alpha-\beta+1)}+\frac{T^{\alpha}}{\Gamma(\alpha+1)}\right.\right. \\
\quad+\frac{a T^{\alpha}}{|1-a| \Gamma(\alpha+1)}+\frac{a T^{\alpha} \Gamma(2-\beta)}{|1-a| \Gamma(\alpha-\beta+1)} \\
\left.\left.\quad+\frac{T^{\alpha} \Gamma(2-\beta)}{\Gamma(\alpha-\beta+1)}\right) \phi_{q}(M)\right] \\
=\frac{1}{2}\left[2 \times \frac{3+\pi}{25 \sqrt{\pi}}-\frac{3+\pi}{25 \sqrt{\pi}} \times\left(\frac{13}{100}\right)^{3}\right] \\
=\frac{3+\pi}{50 \sqrt{\pi}}\left[2-\left(\frac{13}{100}\right)^{3}\right] .
\end{gathered}
$$

Hence, by Theorem 7, BVP (53) has a unique solution for $0 \leq$ $\lambda \leq((3+\pi) / 50 \sqrt{\pi})\left[2-(13 / 100)^{3}\right]$.

\section{Acknowledgments}

This research is supported by Henan Province College Youth Backbone Teacher Funds (2011GGJS-213) and the National Natural Science Foundation of China (11271336).

\section{References}

[1] X. Q. Zhang, "Positive solution for a class of singular semipositone fractional differential equations with integral boundary conditions," Boundary Value Problems, vol. 2012, p. 123, 2012.

[2] G. Chai, "Positive solutions for boundary value problem of fractional differential equation with p-Laplacian operator," Boundary Value Problems, vol. 2012, p. 18, 2012.

[3] Z.-W. Lv, "Positive solutions of m-point boundary value problems for fractional differential equations," Advances in Difference Equations, vol. 2011, Article ID 571804, 13 pages, 2011.

[4] K. S. Miller and B. Ross, An Introduction to the Fractional Calculus and Fractional Differential Equations, John Wiley \& Sons, New York, NY, USA, 1993. 
[5] R. P. Agarwal, V. Lakshmikantham, and J. J. Nieto, "On the concept of solution for fractional differential equations with uncertainty," Nonlinear Analysis. Theory, Methods \& Applications, vol. 72, no. 6, pp. 2859-2862, 2010.

[6] R.-N. Wang, T.-J. Xiao, and J. Liang, "A note on the fractional Cauchy problems with nonlocal initial conditions," Applied Mathematics Letters, vol. 24, no. 8, pp. 1435-1442, 2011.

[7] R.-N. Wang, D.-H. Chen, and T.-J. Xiao, "Abstract fractional Cauchy problems with almost sectorial operators," Journal of Differential Equations, vol. 252, no. 1, pp. 202-235, 2012.

[8] J. Henderson and A. Ouahab, "Impulsive differential inclusions with fractional order," Computers \& Mathematics with Applications, vol. 59, no. 3, pp. 1191-1226, 2010.

[9] V. Lakshmikantham, "Theory of fractional functional differential equations," Nonlinear Analysis. Theory, Methods \& Applications, vol. 69, no. 10, pp. 3337-3343, 2008.

[10] V. Lakshmikantham and A. S. Vatsala, "Basic theory of fractional differential equations," Nonlinear Analysis. Theory, Methods \& Applications, vol. 69, no. 8, pp. 2677-2682, 2008.

[11] C. F. Li, X. N. Luo, and Y. Zhou, "Existence of positive solutions of the boundary value problem for nonlinear fractional differential equations," Computers \& Mathematics with Applications, vol. 59, no. 3, pp. 1363-1375, 2010.

[12] F. Li, "Mild solutions for fractional differential equations with nonlocal conditions," Advances in Difference Equations, vol. 2010, Article ID 287861, 9 pages, 2010.

[13] G. M. Mophou, "Existence and uniqueness of mild solutions to impulsive fractional differential equations," Nonlinear Analysis. Theory, Methods \& Applications, vol. 72, no. 3-4, pp. 1604-1615, 2010.

[14] H. A. H. Salem, "On the fractional order m-point boundary value problem in reflexive Banach spaces and weak topologies," Journal of Computational and Applied Mathematics, vol. 224, no. 2, pp. 565-572, 2009.

[15] R. P. Agarwal and B. Ahmad, "Existence theory for anti-periodic boundary value problems of fractional differential equations and inclusions," Computers \& Mathematics with Applications, vol. 62, no. 3, pp. 1200-1214, 2011.

[16] A. Alsaedi, B. Ahmad, and A. Assolami, "On antiperiodic boundary value problems for higher-order fractional differential equations," Abstract and Applied Analysis, vol. 2012, Article ID 325984, 15 pages, 2012.

[17] B. Ahmad and J. J. Nieto, "Anti-periodic fractional boundary value problems," Computers \& Mathematics with Applications, vol. 62, no. 3, pp. 1150-1156, 2011.

[18] Z. H. Liu, "Anti-periodic solutions to nonlinear evolution equations," Journal of Functional Analysis, vol. 258, no. 6, pp. 2026-2033, 2010.

[19] G. Wang, B. Ahmad, and L. Zhang, "Impulsive anti-periodic boundary value problem for nonlinear differential equations of fractional order," Nonlinear Analysis. Theory, Methods \& Applications, vol. 74, no. 3, pp. 792-804, 2011.

[20] S. Q. Zhang, "Positive solutions for boundary-value problems of nonlinear fractional differential equations," Electronic Journal of Differential Equations, vol. 36, pp. 1-12, 2006.

[21] A. A. Kilbas, H. M. Srivastava, and J. J. Trujjllo, "Theory and applications of fractional differential equations," in NorthHolland Mathematics Studies, vol. 204, Elsevier, Amsterdam, The Netherlands, 2006.

[22] Z. Bai and H. Lü, "Positive solutions for boundary value problem of nonlinear fractional differential equation," Journal of Mathematical Analysis and Applications, vol. 311, no. 2, pp. 495-505, 2005. 


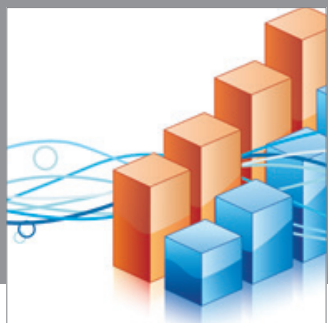

Advances in

Operations Research

mansans

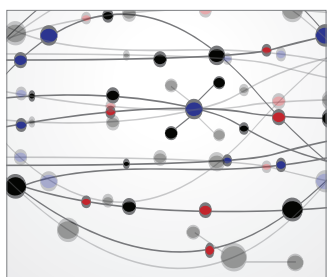

The Scientific World Journal
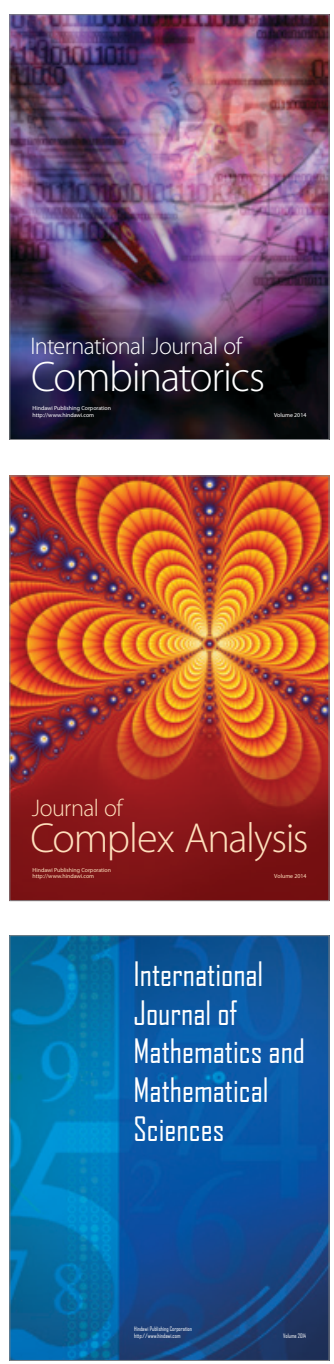
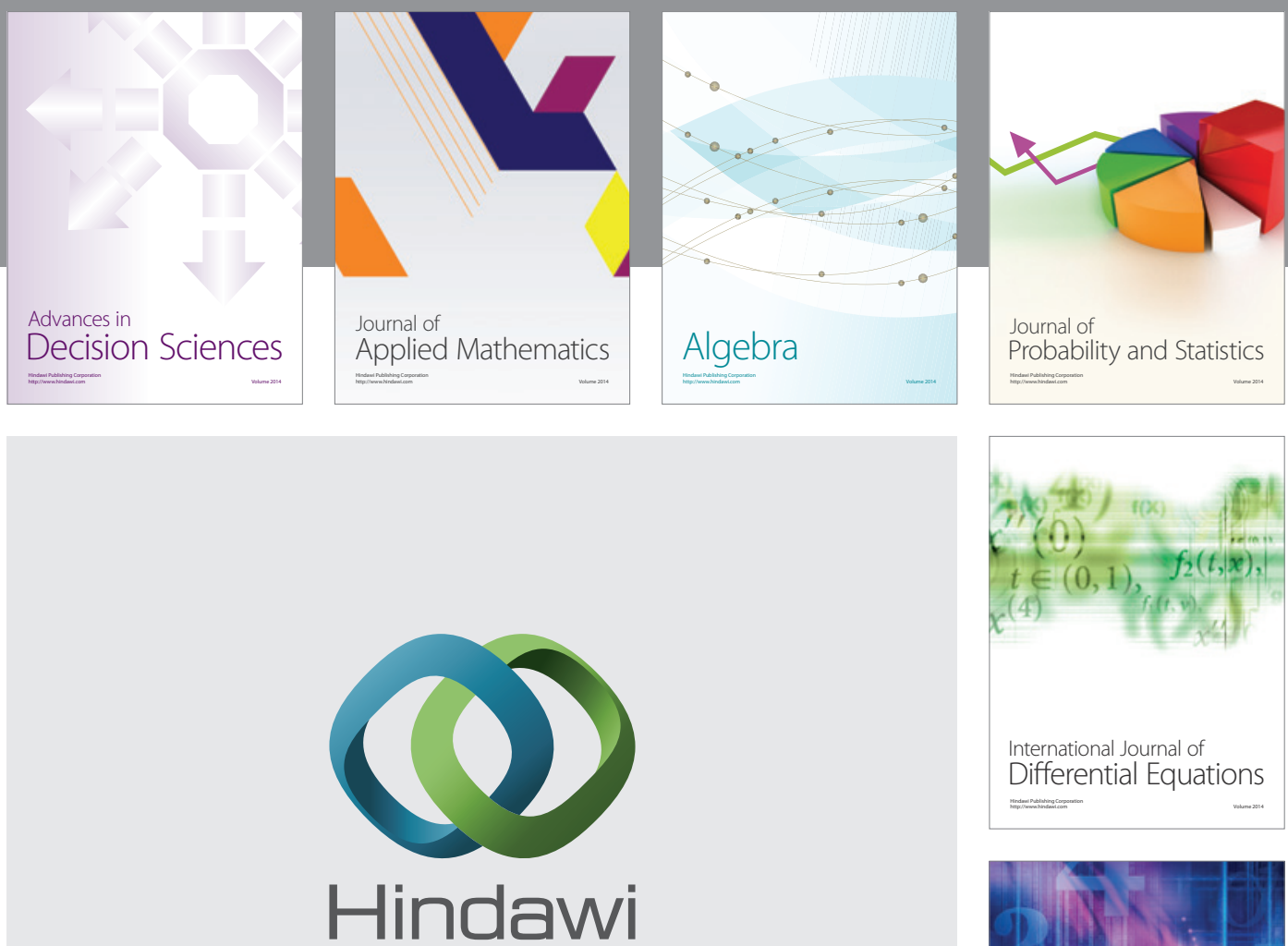

Submit your manuscripts at http://www.hindawi.com
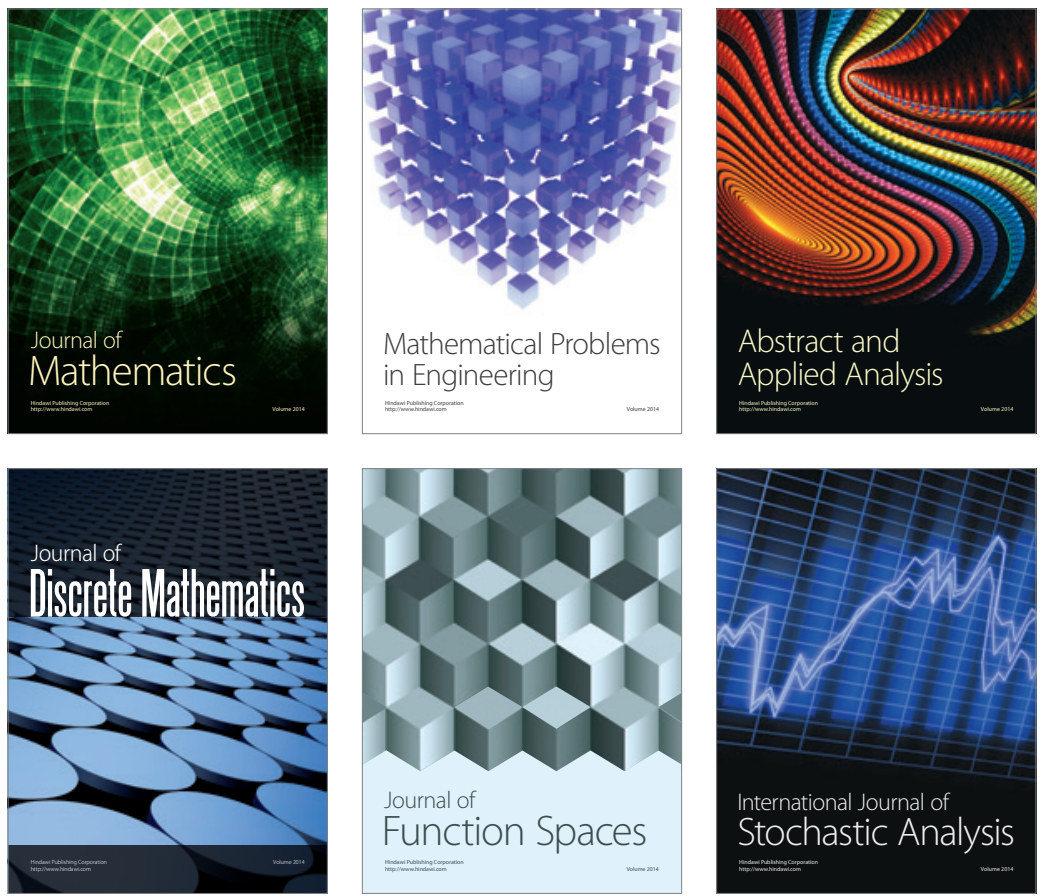

Journal of

Function Spaces

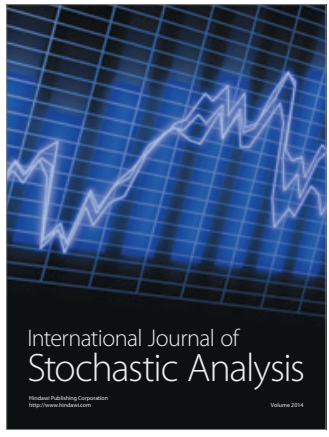

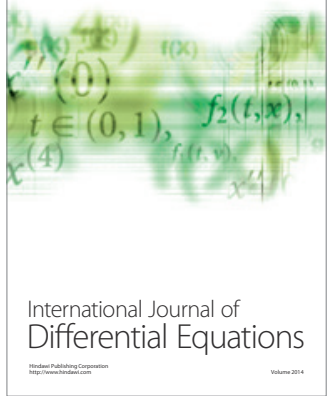
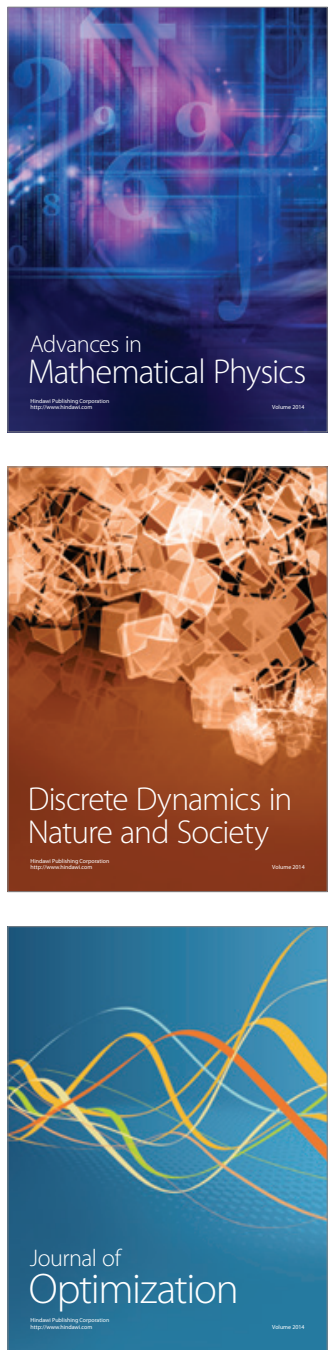